\title{
DISSECÇÃO DE AORTA: UM CASO DE SUCESSO NA MEDICINA
}

\author{
AORTIC DISSECTION: A SUCESSFUL CASE IN MEDICINE
}

\author{
Anne Louyse Andrade Lira ${ }^{1}$ \\ Raimunda Leite de Alencar Neta ${ }^{2}$ \\ Welington Antônio Silva ${ }^{3}$ \\ Ankilma do Nascimento Andrade Feitosa ${ }^{4}$ \\ Renata Livia Silva Fonsêca Moreira de Medeiros ${ }^{5}$ \\ Macerlane de Lira Silva ${ }^{6}$
}

RESUMO: Objetivo: Descrever um caso de dissecção de aorta diagnosticado e tratado com sucesso pela equipe médica, enfatizando a importância do diagnóstico precoce. Metodologia: Trata-se de uma pesquisa do tipo estudo de caso, descritiva, com abordagem qualitativa, sendo realizada com um paciente procedente $e$ residente da cidade de São José de Piranhas-PB. Para a coleta de dados, realizouse uma entrevista, que ocorreu entre dezembro de 2018 e março de 2019. Como instrumento, utilizou-se um questionário semiestruturado, contendo dados sociodemográficos e específicos, no intuito de realizar os objetivos do estudo. A pesquisa obedeceu aos princípios éticos de acordo com a resolução 510/16 do Conselho Nacional de Saúde, que trata de pesquisa e testes envolvendo seres humanos. O projeto foi submetido ao Comitê de Ética em Pesquisa da Faculdade Santa Maria. Diante avaliação e aprovação pelo órgão mencionado, solicitou-se a

\footnotetext{
1 Discente do curso de Bacharelado em Medicina pela Faculdade Santa Maria (FSM).

2 Discente do curso de Bacharelado em Enfermagem pela Faculdade Santa Maria (FSM).

3 Docente do curso de Bacharelado em Medicina pela Faculdade Santa Maria (FSM), Médico Cardiologista formado pela Universidade Federal do Pernambuco - UFPE, Residência em Clínica Médica pelo Hospital Geral Dr César Calls e Residência em Cardiologia pelo Hospital Dr Carlos Alberto Studart Gomes.

${ }^{4}$ Docente da Faculdade Santa Maria - FSM. Graduada em Enfermagem - FAZER, Licenciada em Enfermagem - UFPB, Especialista em Auditoria em Serviços de Saúde - FACISA, Mestre em enfermagem - UFPB, Doutora em Ciências da Saúde pela Faculdade de Medicina do ABC - FMABC.

${ }^{5}$ Docente da Faculdade Santa Maria - FSM. Enfermeira pela Universidade Federal da Paraíba UFPB. Especialisa em Saúde Pública pela FACISA. Mestre em Enfermagem pela Universidade Federal da Paraíba - UFPB. Doutora em Pesquisa em Cirurgia pela Faculdade de Ciências Médicas Santa Casa de São Paulo - FCMSP.

6 Docente da Faculdade Santa Maria - FSM. Graduação em Enfermagem pela FSM Cajazeiras, especialização em política e gestão do cuidado com ênfase no apoio matricial pela UFPB e mestrado em Saúde Coletiva pela UNISANTOS.
} 
participação do paciente, o qual recebeu esclarecimentos sobre os objetivos e métodos da pesquisa, por meio de informações contidas no Termo de Consentimento Livre e Esclarecido, e após assinatura deste. Resultados: A amostra tratou-se de um homem, pardo, ensino superior completo, com 53 anos de idade, e que foi diagnosticado com dissecção da aorta aos 50 anos após ecocardiograma e angiotomografia torácica e abdominal, apresentando dor torácica de forte intensidade. Após a implantação da endoprótese em aorta torácica, verificou-se ausência de sequelas após o tratamento cirúrgico, e o procedimento interferiu de maneira positiva na qualidade de vida do paciente. Conclusão: As informações acerca dessa temática mostram a relevância para que profissionais da saúde realizem o manejo clínico de pacientes como relatado, desde a sintomatologia inicial até a confirmação do diagnóstico e tratamento. Além disso, o presente estudo pode colaborar positivamente para que novas publicações sobre o assunto sejam realizadas futuramente.

Palavras chave: Aorta. Diagnóstico. Dissecção.

ABSTRACT: Objective: To describe a case of aortic dissection successfully diagnosed and treated by the medical team, with emphasis on the importance of early diagnosis. Methodology: This was a descriptive case study, with a qualitative approach, conducted with a patient coming from the city of São José de Piranhas $P B$. For data collection, an interview was held, which took place from December 2018 to March 2019. The instrument used was a questionnaire, divided into sociodemographic and specific data, in order to achieve the study objectives. The research obeyed the ethical principles in accordance with Resolution 510/16 of the National Health Council, which deals with research and tests involving human beings. The project was submitted to the Research Ethics Committee of Santa Maria College. Upon evaluation and approval by the aforementioned body, the patient was invited to participate, receiving clarifications on the research objectives and methods, through information contained in the Informed Consent Form, and after signing it. Results: the sample was composed of a man, pardo, complete higher education, aged 53 years, and was diagnosed with aortic dissection at 50 years old after echocardiogram and thoracic and abdominal angiography, presenting severe chest pain. After implantation of the stent in the thoracic aorta, there was absence of sequelae after surgical treatment, and the procedure positively interfered with his quality of life. Conclusion: The information on this theme shows the relevance for health professionals perform the clinical management of patients as reported, from the initial symptomatology to the confirmation of diagnosis and treatment. In addition, the present study may contribute positively to further publications on the subject in the future.

Keywords: Aorta. Diagnosis. Dissection. 


\section{INTRODUÇÃO}

A doença cardiovascular é a principal causa de mortalidade nos países em desenvolvimento e na maioria dos países desenvolvidos. Apesar do declínio da mortalidade por doenças cardiovasculares no mundo desenvolvido (infarto do miocárdio e cerebral), nos países em desenvolvimento, essa curva ainda é ascendente e acentuada, apresentando-se, pelo menos, 2,8 vezes maior quando comparada aos países desenvolvidos, principalmente devido às alterações do estilo de vida impostas pela industrialização e urbanização, associadas ao aumento da expectativa de vida, hábitos alimentares inadequados, e ao tabagismo (DIAS et al., 2013).

Pérez et al. (2012) afirmam que a dissecção aórtica é uma doença da camada média do vaso em que o fluxo sanguíneo ocorre entre as camadas média e íntima, apresentando-se como a emergência mais comum da aorta por ser uma doença grave de alta mortalidade. Michaelis (2017) complementa que há diversos fatores que podem levar à degeneração da camada média e ao surgimento da dissecção, entre eles aterosclerose, hipertensão, tabagismo, sexo masculino e arteriopatias inflamatórias; porém, para Formiga e Fanelli (2015), a hipertensão é o principal fator predisponente para a dissecção da aorta.

Para Michaelis (2017), avalia-se a dissecção da aorta através da classificação de DeBakey ou de Stanford. A primeira classifica de acordo com o acometimento da aorta ascendente (tipo A) ou da aorta descendente (tipo B); a classificação de DeBakey refere-se ao acometimento da aorta ascendente, dividida em tipo I (acomete desde a artéria ascendente até a descendente), tipo II (restrita à ascendente) e tipo III (acomete a descendente).

A dissecção de aorta tipo A é o evento cardiovascular de maior mortalidade aguda quando não tratado em tempo, visto que, nesta fase, os pacientes evoluem para morte súbita em $3 \%$ dos casos e, na ausência de atendimento adequado imediato, a mortalidade pode ter um aumento de $1 \%$ a cada hora nas primeiras 24 
horas, sendo que $80 \%$ morrem nas primeiras duas semanas e $95 \%$, no decorrer de um ano (PONTES et al., 2012).

O principal sintoma encontrado para o diagnóstico de dissecção de aorta é a dor torácica aguda com irradiação para o dorso, associado a quadro hipertensivo, bradicardia e síncope, o que o torna sempre complexo, seja pela baixa incidência ou procura de serviços médicos, seja pela apresentação inespecífica, com primeiro diagnóstico equivocado em 38\% dos casos na avaliação inicial (MICHAELIS, 2017).

Sua resolução é determinada pelo tipo e extensão da dissecção e pela presença de complicações associadas, como acidente vascular cerebral isquêmico, envolvimento da raiz da aorta e valva aórtica, derrame pericárdio e abdômen agudo vascular ou isquemia de membros inferiores. Para que haja um melhor prognóstico, o diagnóstico e o tratamento precoces são de extrema importância (DE SOUZA JUNIOR et al., 2012).

A incidência para dissecções agudas da aorta é de 10 a 20 por 1.000 .000 de pessoas / ano. A idade média no diagnóstico varia de 59 a 69 anos, com predominância de homens com uma frequência de duas a quatro vezes maiores do que em mulheres (NOVAES et al., 2013). Mais de 47 mil indivíduos por ano morrem vítimas das doenças da aorta, mais do que câncer de mama, AIDS, homicídios ou acidentes automobilísticos, fazendo da doença aórtica uma epidemia silenciosa (ISSA, 2012).

Sousa Júnior (2012) classifica quanto ao quadro clínico da doença que $85 \%$ dos pacientes queixam-se de dor com início súbito, sendo que $82 \%$ dessa dor está localizada no tórax e 43\%, no dorso. Além disso, nos pacientes, pode ser observado déficit neurológico em 14\%, síncope em $17 \%$, assimetria de pulso radial em $26 \%$ e hipotensão, choque e tamponamento cardíaco em $22 \%$ dos casos.

As dissecções da aorta ascendente e os aneurismas que necessitam de tratamento cirúrgico permanecem como desafios para os cirurgiões cardiovasculares, devido à anatomia desta região, frequentemente com envolvimento da valva aórtica, dos seios de Valsalva, dos óstios coronares, da porção tubular reta de aorta, talvez estendendo-se até o arco aórtico e seus ramos (NOVAES et al., 2013). 
Nesse contexto, a justificativa para realização desse trabalho baseia-se no fato de que o diagnóstico de dissecção de aorta é complexo devido à apresentação inespecífica do quadro, porém é necessário que ocorra o mais rápido possível para que o tratamento seja realizado possibilitando um melhor prognóstico e diminuição da mortalidade. Sendo assim, serão aprofundados os conhecimentos acerca do tema, visto que o diagnóstico precoce é essencial para a realização da conduta terapêutica mais adequada e eficaz na redução de complicações.

Dessa forma, espera-se que informações acerca do tema sejam elucidadas para os profissionais da saúde, e que revelem como foi realizado o manejo clínico desse paciente, desde a sintomatologia inicial até a confirmação do diagnóstico e tratamento. Além disso, também é esperado que o estudo colabore para novas publicações sobre o assunto.

\section{METODOLOGIA}

Trata-se de um estudo de caso, descritivo, com abordagem qualitativa, realizado no município de São José de Piranhas-PB. Formado por população e a amostra análogas, incluindo um adulto com diagnóstico de dissecção de aorta.

Foram selecionados pacientes que possuíam Dissecção de Aorta, documentos que comprovassem informações sobre a história clínica do paciente desde o diagnóstico até a finalização da coleta de dados e que assinaram o Termo de Consentimento Livre e Esclarecido (TCLE), aceitando participar da pesquisa.

Visto isso, foram excluídos os participantes que não possuíam diagnóstico definido da doença, assim como os que não portavam nenhum registro que possibilitasse a realização do estudo.

Após a aceitação do projeto submetido ao Comitê de Ética em Pesquisa (CEP) da Faculdade Santa Maria (FSM), foi realizado o agendamento da entrevista para a coleta de informações. Na coleta de dados, solicitou-se anuência do paciente através do TCLE. 
A coleta de dados ocorreu no período de dezembro de 2018 a março de 2019 após emissão de parecer pelo CEP da FSM e autorização do participante do estudo, diretamente com o paciente que tem dissecção de aorta, através de uma entrevista previamente agendada de acordo com sua disponibilidade.

A coleta de dados ocorreu por meio de uma entrevista semiestruturada, composta por 13 questões, divididas em duas categorias: dados epidemiológicos e dados específicos. Além disso, foram obtidos dados presentes em resultados de exames e relatórios que se encontravam com o paciente, pertinentes aos objetivos da pesquisa.

A análise dos dados ocorreu de maneira qualitativa através de uma análise sintomatológica inicial do paciente, exames solicitados, como se deu o diagnóstico precoce e conduta realizada.

\section{RESULTADOS}

O estudo foi realizado com um homem, pardo, ensino superior completo, com 53 anos de idade, diagnosticado com dissecção de aorta aos 50 anos de idade, hipertenso, que teve como sintomatologia uma dor torácica de forte intensidade que irradiava para o dorso. Foi conduzido por seus familiares de São José de PiranhasPB até a Unidade de Pronto Atendimento (UPA) em Cajazeiras - PB, onde foi encaminhado para o Hospital Regional de Cajazeiras (HRC). No local, os profissionais efetuaram o protocolo para Infarto Agudo do Miocárdio, por acreditarem que se tratava desse diagnóstico.

Após realizar estabilização e encaminhar o paciente para o cardiologista, o paciente recebeu alta. Ao chegar a sua residência, ele voltou a apresentar a sintomatologia, associada a náuseas, episódios de êmese e diarreia. No dia seguinte, foi realizada uma consulta com um cardiologista, que, devido à sintomatologia e à realização de um ecocardiograma, transferiu-o com urgência para Barbalha-CE, onde foi realizada uma angiotomografia de tórax e abdome, a qual evidenciou dissecção de aorta total. Após isso, foi realizada cirurgia de reconstrução 
do arco da aorta, sendo necessária realização de uma nova cirurgia para colocação de endoprótese torácica.

Durante a realização da entrevista para levantamento dos dados, o paciente tinha realizado eletrocardiograma, ecocardiograma, angiotomografia torácica e abdominal, além de exames laboratoriais.

Como tratamentos, foram realizados: intervenção cirúrgica com a implantação da endoprótese em aorta torácica e utilização de medicamentos específicos para o controle da hipertensão e anticoagulantes.

Após o tratamento cirúrgico, foi verificada ausência de sequelas, apresentando melhoras na qualidade de vida do paciente submetido à dissecção da aorta.

Com base nos hábitos realizados pelo referido paciente para se obter uma vida saudável, constatou-se uma mudança radical em seu estilo de vida, no qual inclui: alimentação saudável, visita frequente ao cardiologista, exames de rotina realizados a cada seis meses e uso de medicamentos contínuos.

\section{DISCUSSÃO}

Baseando-se em Oliveira (2017), o aparelho circulatório pode ser visto como um exemplo de sensibilidade humana, uma vez que, por ser composto de um sistema vascular complexo, ele exerce basicamente a função de garantir a correta circulação sanguínea a diversos órgãos e tecidos do corpo humano, trabalhando de forma eficiente a uma temperatura média de $37^{\circ} \mathrm{C}$. Para o autor, as doenças do aparelho circulatório podem ser vistas como uma das principais causas de óbitos em países desenvolvidos e subdesenvolvidos.

Mediante os resultados encontrados, ressalta-se que as cirurgias cardíacas constituem-se em procedimentos relacionados a várias complicações pósoperatórias. Nesse âmbito, Buono et al. (2015) citam que é de fundamental importância o envolvimento de uma equipe multidisciplinar, a fim de proporcionar 
uma recuperação global e melhorar a qualidade de vida de pacientes submetidos a tais procedimentos, conforme mostrado na referida pesquisa.

As cirurgias cardíacas são de grande porte e difundidas mundialmente. Dentre elas, destaca-se a dissecção da aorta, consistindo em um tratamento invasivo, que visa a promover uma melhor qualidade de vida. A cirurgia cardíaca é indicada em casos em que os resultados não foram satisfatórios nas seguintes terapêuticas: medicação trombolítica e angioplastia primária com deterioração clínica do paciente, uma vez que o diagnóstico de dissecção de aorta é complexo devido à apresentação inespecífica do quadro cardíaco (FERREIRA et al., 2013).

Sabe-se que a correção cirúrgica é uma alternativa para muitas doenças do sistema cardiovascular. Com base em Santana et al. (2013, p. 12), "uma série de técnicas e tipos de incisões é utilizada para a realização dos procedimentos que incluem dissecção da aorta, reparos e trocas das válvulas cardíacas e correção de patologias cardíacas congênitas".

No trabalho de Benetti, Araújo e Santos (2016), a cirurgia de dissecção da aorta é considerada o padrão ouro para o tratamento da doença coronariana e emergências complexas. Sabe-se que os cirurgiões cardiovasculares deparam-se com um número cada vez maior de pacientes de alto risco, porém, apesar dos avanços nas técnicas cirúrgicas e na proteção miocárdica, a dissecção torna-se um recurso promissor.

Nessa perspectiva, juntamente ao avanço das técnicas cirúrgicas, o desenvolvimento da cardiologia intervencionista tem causado o aumento das opções terapêuticas para o tratamento da dissecção da aorta. Com o rápido progresso e experiência adquirida ao longo dos anos, a dissecção passou a ser uma alternativa segura e menos invasiva (SILVA; LUIZ; PEREIRA, 2015).

Ainda corroborando os atores supramencionados, nos casos de dissecções envolvendo a aorta ascendente, a intervenção cirúrgica (aneurismectomia) deve ser imediata e tem por objetivos evitar ruptura e morte por tamponamento cardíaco, corrigir a regurgitação aórtica, evitar a isquemia miocárdica e redirecionar o fluxo sanguíneo aos ramos supra-aórticos e à aorta descendente (SANTANA et al., 2013). Dutra et al. (2016) ainda complementam que alguns dos principais fatores de risco para ruptura de aneurismas são: dissecção de diâmetro maior que cinco 
centímetros, evolução no crescimento do aneurisma, tabagismo, doença pulmonar obstrutiva crônica, hipertensão arterial sistêmica e doenças degenerativas da aorta.

Os resultados aqui encontrados corroboram Gomes e Gomes (2012), os quais mencionam que inúmeras complicações podem elevar a morbidade e a mortalidade dos pacientes no período pós-operatório, aumentando o risco inerente ao procedimento. Os autores citam ainda que a dissecção cria um novo percurso para o fluxo sanguíneo e, uma vez que o volume e a pressão do sangue são restaurados pelo procedimento cirúrgico, aliviam-se os sintomas, tais como dispneia ao esforço físico e angina, decorrentes da má nutrição e hipóxia.

Fato semelhante foi encontrado por Silva, Luiz e Pereira (2015), onde os autores relatam que algumas limitações impedem a revascularização apenas com enxertos arteriais, incluindo a dificuldade no procedimento de dissecção sem danos irreversíveis que comprometam o resultado cirúrgico e a pouca disponibilidade de artérias para a completa revascularização pós dissecção da aorta.

Em consonância com os autores citados anteriormente, o aumento das propriedades espásticas desses vasos compromete a perfusão do coração, com consequentes complicações isquêmicas no pré e pós-operatório imediatos, aumentando a mortalidade relacionada ao procedimento (SILVA; LUIZ; PEREIRA, 2015).

Apesar disso, a maioria absoluta dos pacientes encaminhados à cirurgia de dissecção necessita de pelo menos um enxerto com a veia safena, considerado um enxerto livre. Contudo, conforme os achados do referido estudo, Mendes et al. (2016, p. 70) mencionam que "a dissecção da aorta, quando realizada dentro das condições ideais garante a melhoria dos sintomas decorrentes da insuficiência cardíaca, bem como o aumento da sobrevida e, consequentemente, proporciona um ganho na qualidade de vida do paciente".

A reabilitação cardiovascular pode ser caracterizada como o processo de restauração vital, compatível com a capacidade funcional do coração daqueles indivíduos que já apresentaram manifestações prévias de emergências hipertensivas, destacando a importância do autocuidado para a recuperação funcional e a melhora na qualidade de vida (PIMENTEL et al., 2013). 
Mediante os resultados encontrados, é importante frisar ainda que "o processo de desenvolvimento e manutenção pós dissecção da aorta visa a obter um nível desejável das condições físicas, mentais e sociais, assegura o retorno do paciente a uma vida ativa e produtiva da melhor maneira possível" (SILVA, 2016, p. 44).

A reabilitação cardiovascular pós dissecção da aorta é indicada quando o paciente encontra-se em fase estável. Conforme citado por Dantas e Torranti (2012), quando o processo de melhoras pós dissecção está sendo devidamente medicado e não apresenta sinais e sintomas da doença, inicia-se o programa de reabilitação cardíaca, onde o paciente deverá ser submetido a várias avaliações e a uma série de testes para que os profissionais conheçam bem o paciente e seu quadro clínico.

O consenso de reabilitação cardiovascular caracteriza a reabilitação como a somatória das atividades para garantir melhores condições físicas e sociais possíveis, de modo que os pacientes possam, com seus próprios esforços, recuperar uma vida ativa e produtiva (SOCIEDADE BRASILEIRA DE CARDIOLOGIA, 2014).

A avaliação médica deverá ser bastante rigorosa, investigando toda a história atual e pregressa do paciente, além de seus antecedentes familiares. Uma boa anamnese pode revelar informações importantes que poderão contribuir substancialmente para o tratamento, que deve adotar uma abordagem em equipe, onde 0 médico tem 0 papel fundamental de monitorizar a tolerância à farmacoterapia, à atividade, preparar o paciente para alta, orientá-lo para que reconheça os sintomas adversos decorrentes da atividade, dando também apoio emocional e colaborando com outros integrantes da equipe multidisciplinar (SANTANA et al., 2013).

O processo de retorno da qualidade de vida tem como objetivo mostrar como medidas não farmacológicas no tratamento do paciente com doença pós dissecção, oferece reabilitação de forma segura, associado a uma abordagem multidisciplinar com grande enfoque na educação do paciente, principalmente em relação à mudança do estilo de vida como prática para a vida inteira.

A multidisciplinaridade na reabilitação cardíaca é de fundamental importância para que o indivíduo retome suas atividades rotineiras o mais rápido possível e 
também para que se conscientize sobre mudanças nos hábitos de vida, tais como alimentação saudável, a prática de exercícios físicos, além de evitar tabagismo, álcool e gorduras em excesso (DUTRA et al., 2016).

\section{CONCLUSÃO}

Com os resultados obtidos neste estudo, pode-se supor que alterações do sistema cardiovascular podem ocasionar a dissecção da aorta.

Elencou-se que a intervenção médica pode auxiliar os pacientes no processo de reabilitação e melhoria da qualidade de vida, fazendo orientações voltadas para o autocuidado, alimentação saudável, mudança nos hábitos de vida, prática de exercícios físicos, evitar tabagismo, álcool e gorduras em excesso, bem como para aprenderem a lidar com todos os aspectos emocionais, sociais e físicos envolvidos em uma cirurgia cardíaca.

É importante ainda esclarecer que, embora os objetivos tenham sido alcançados, não se pretendeu, com essa pesquisa, encerrar as discussões ou tirar conclusões definitivas sobre o tema, ao contrário, pretendeu-se demonstrar a relevância da discussão, em âmbitos acadêmicos e profissionais, acerca da medição e da avaliação destas variáveis, através da percepção dos pacientes, e no contexto do processo de tomada de decisões clínicas e na determinação dos benefícios terapêuticos. 


\section{REFERÊNCIAS BIBLIOGRÁFICAS}

ARAÚJO, C. et al. Estudo de Caso. Métodos de Investigação em Educação. Instituto de Educação e Psicologia, Universidade do Minho, 2008. Disponível em <http://grupo4te.com.sapo.pt/estudo_caso.pdf>. Acesso em: 07 nov. 2019.

AUDI, C. A. F. et al. Fatores de risco para doenças cardiovasculares em servidores de instituição prisional: estudo transversal. Rev. Epidemiol. Serv. Saúde. v. 25, n. 2, p. 301-310, 2016. Disponível em: <http://www.scielo.br/scielo.php?script=sci_arttext\&pid =S223796222016000200301\&lng=en\&nrm=iso $>$. doi: https://dx.doi.org/10.5123/s167949742016000200009.

BENETTI, M.; ARAÚJO, C.L.P.; SANTOS, R.Z. Aptidão Cardiorrespiratória e Qualidade de Vida Pós-Enfarte em Diferentes Intensidades de Exercício. Arq. Bras. Cardiol. v. 95, n. 3, p. 399404, 2016. Disponível em:<http://www.scielo.br/pdf/abc/2d/aop08810> Acesso em: 25 nov. 2019. https://dx.doi.org/10.1590/S0066-782X2010005000089.

BUONO, H. C. D. et al. Fisiologia cardíaca, valvopatias e a atuação do nutricionista. Rev. Saúde em Foco. n.7, p. 197-202, 2015. Disponível em: <http://portal.unisepe.com.br/unifia/wpcontent/uploads/sites/10001/2018/06/23fisiolo_cardiaca.pdf>.

CESAR, L. A. et al. Diretriz de Doença Coronária Estável. Arquivos Brasileiros de Cardiologia. v. 103 , n. 2, supl. 2, p. 1-59, 2014. Disponível em: <http://publicacoes.cardiol.br/2014/diretrizes/2014/Diretriz\%20de\%20Doen\%C3\%A7a\%20Coron \%C3\%A1ria\%20Est\%C3\%A1vel.pdf >.

DANTAS, R. A. S.; TORRATI, F. G. Circulação extracorpórea e complicações no período pósoperatório imediato de cirurgias cardíacas. Acta paul. enferm. v. 25, n. 3, p. 340-345, 2012. Disponível em: <http://www.scielo.br/scielo.php?script=sci_arttext\&pid=S0103$21002012000300004 \&$ Ing=en\&nrm=iso >. https://dx.doi.org/10.1590/S0103-21002012000300004.

DIAS, R. R. et al. Impacto da mortalidade da doença da aorta torácica no estado de São Paulo no período de 1998 a 2007. Arq. Bras. Cardiol. v. 101, n. 6, p. 528-535, 2013. Disponível em: <http://www.scielo.br/scielo.php?script=scinrm=iso>. $\quad$ Acesso em: 24 out. 2019. https://dx.doi.org/10.5935/abc.20130203.

DUTRA, D. D. et al. Doenças cardiovasculares e fatores associados em adultos e idosos cadastrados em uma unidade básica de saúde. Rev. Pesq. Cuid. Fundam. v. 8, n. 2, p. 4501$\begin{array}{lll}4509, & 2016 . & \text { Disponível }\end{array}$ <http://seer.unirio.br/index.php/cuidadofundamental/article/view/4787/pdf_1906>. http://dx.doi.org/10.9789/2175-5361.2016.v8i2.4501-4509.

FERREIRA, A. G. et al. A doença arterial coronariana e o envelhecimento populacional: como enfrentar esse desafio? Rev. HUPE. v. 12, supl. 1, p.13-24, 2013. Disponível em: <https://www.e-publicacoes.uerj.br/index.php/revistahupe/article/view/7079>.

https://doi.org/10.12957/rhupe.2013.7079.

FORMIGA, M. N. da C.; FANELLI, M. F. Aortic dissection during antiangiogenic therapy with sunitinib. A case report. São Paulo Med. J. v. 133, n. 3, p. 275-277, 2015. Disponível em: <http://www.scielo.br/scielo.php?script=sci_arttext\&pid=S1516-31802015000300275>. Acesso em: 24 out. 2019. https://dx.doi.org/10.1590/1516-3180.2013.7380002.

GIL, A.C. Como elaborar projetos de pesquisa. 4. ed. São Paulo: Atlas, 2009. 
GOMES, W.J.; GOMES, O.M. Anatomia do coração aplicada à clínica. Livro-texto da Sociedade Brasileira de Cardiologia. Barueri, SP: Manole. 2012.

INSTITUTO BRASILEIRO DE GEOGRAFIA E ESTATÍSTICA (IBGE). IBGE cidades. Paraíba São José de Piranhas. 2017. Disponível em <https://www.ibge.gov.br/geocienciasnovoportal/organizacao-do-territorio/estrutura-territorial/15761-areas-dos-

municipios.html?t=destaques\&c=2514503>. Acesso em 07 de nov. 2019.

ISSA, M. Variáveis prognósticas de evolução hospitalar e no longo prazo de pacientes portadores de dissecção crônica de aorta tipo A de Stanford e aneurisma de aorta ascendente, submetidos a procedimento cirúrgico. 2012. Tese (Doutorado em Medicina/Tecnologia e Intervenção em Cardiologia) - Instituto Dante Pazzanese de Cardiologia, Universidade de São Paulo, São Paulo, 2012. doi:10.11606/T.98.2012.tde-28062012-103725. Acesso em24 Out. 2019.

MENDES, M. J. F. L.; ALVES, J. G. B.; ALVES, A. V.; SIQUEIRA, P. P.; FREIRE, E. F. C. Associação de fatores de risco para doenças cardiovasculares em adolescentes e seus pais. Rev. Bra. Saude Mater. Infant. v. 6, supl. 1, p. 49-54, 2016. Disponível em: < http://www.scielo.br/scielo.php?script=sci_arttext\&pid=S1519-

$38292006000500007 \&$ Ing=en\&nrm=iso >. https://dx.doi.org/10.1590/S1519-38292006000500007.

MICHAELIS, W. et al. Dissecção aórtica de tipo B de Stanford: relato de caso e revisão de literatura. J. vasc. bras. v. 16 , n. $3, \quad$ p. 252-257, 2017. Disponível em $<$ http://www.scielo.br/scielo.php?script=sci_arttext\&pid=S1677-

54492017000300252\&lng=en\&nrm=iso>. Acesso em: 24 out. 2019. https://dx.doi.org/10.1590/1677-5449.000117.

NOVAES, F. R. et al. Results of Castro Bernardes intraluminal ring in surgery for ascending aortic aneurysms and dissections. Rev Bras Cir Cardiovasc. v. 28, n. 2, p. 176-182, 2013. Disponível em: <http://www.scielo.br/scielo.php0102>. Acesso em: 24 out. 2019.

OLIVEIRA, M. A. P. de. Revascularização miocárdica híbrida versus cirúrgica em pacientes com doença aterosclerótica coronária multiarterial: estudo clínico prospectivo randomizado. 2017. 112f. Tese (Doutorado em Ciências) - Faculdade de Medicina da Universidade de São Paulo. Programa de Cirurgia Torácica e Cardiovascular. São Paulo, 2017.

PÉREZ, L. P. Disección del arco aórtico. Rev. electrón. Dr. Zoilo E. MarinelloVidaurreta. v. 37, ก. 12, 2012.

PIMENTEL, J. F. et al. Qualidade de vida em pacientes pós-operatórios de cirurgia cardíaca. Rev. SBPH. v. 16, n. 2, p.120-136, 2013. Disponível em: <http://pepsic.bvsalud.org/scielo.php?script=sci_arttext\&pid=S1516-

08582013000200009\&lng=pt\&nrm=iso>.

PRESTES, M. L. M. A. Pesquisa e a Construção do Conhecimento Científico: do planejamento aos textos, da escola à academia. 2 ed. São Paulo: Rêspel, 2003.

PONTES, J. C. D. V. et al. Correção endovascular de aneurisma de aorta abdominal em complicação tardia de dissecção de aorta tipo A. Rev. Bras. Cir. Cardiovasc. v. 27, n. 4, p. 645647, 2012. Disponível em: <http://www.scielo.br/scielo.php?script=sci_arttext\&nrm=iso>. Acesso em: 24 out. 2019. https://dx.doi.org/10.5935/1678-9741.20120108.

RICHARDSON, R. J. Pesquisa Social: Métodos e Técnicas. 3ed. São Paulo: Atlas, 2007.

SANTANA, G. R. et al. Avaliação da Qualidade de Vida de Pacientes Submetidos à Cirurgia Cardíaca. Estado de Sergipe. Caderno de Graduação-Ciências Biológicas e da Saúde. v.1, n. $\begin{array}{llllll}17, & \text { p. } & 113-124, & 2013 . & \text { Disponível }\end{array}$ 
<https://periodicos.set.edu.br/index.php/cadernobiologicas/article/view/1006/541>.

SILVA, A. J. M. Estudo de risco cardiovascular: uma proposta do uso de mieloperoxidase sérica e avaliação da pressão intracraniana. 2016. 68 f. Dissertação (Mestrado em Ciências Biomédicas) - Universidade Estadual de Ponta Grossa. Ponta Grossa-PR, 2016.

SILVA, S. M.; LUIZ, R. R.; PEREIRA, R. A. Fatores de risco e proteção para doenças cardiovasculares em adultos de Cuiabá, Mato Grosso, Brasil. Rev. Bras. Epidemiol. v. 18, n. 2 , p. 425-438, 2015. Disponível em: <http://www.scielo.br/scielo.php?script=sci_arttext\&pid=S1415790X2015000200425\&lng=en\&nrm=iso $>$. https://dx.doi.org/10.1590/1980-5497201500020011.

SOUZA JUNIOR, E. B. de et al. Dissecção de aorta: desafio diagnóstico. Arq Med Hosp Fac Cienc Med Santa Casa São Paulo. v. 57, p. 139-142, 2012. Disponível em <http://www.fcmscsp.edu.br/files/RC15-Disseccao.pdf>. Acesso em: 24 out. 2019.

ROCHA, S. B. et al. Variáveis cardiorrespiratórias e expansibilidade torácica antes e após o uso do incentivador respiratório no pós operatório de revascularização do miocárdio. Saúde Rev. v. 13, n. 33, p. 47-54, 2013. Disponível em: <https://www.metodista.br/revistas/revistasunimep/index.php/sr/article/viewFile/1078/1182>. http://dx.doi.org/10.15600/22381244/sr.v13n33p47-54. 